\title{
Primary care and the transgender population: Supporting providers through training and education
}

\author{
Katie Ross
}

\section{Brock University}

Canada is home to a very diverse population, which presents both challenges and opportunities for improvement in policy and primary care. One minority group that has gained increasing attention in the medical community over the past decade is the transgender population. Transgender is a term that refers to any individual who identifies differently than the sex that they were assigned at birth, making this an umbrella term encompassing many other trans-related identities such as transsexual, trans man, trans woman, and transitioned (to name a few). Although there is insufficient data regarding the proportion of the population that is transgender in Canada, it has been reported that the prevalence of gender dysphoria for natal adult males is between $0.005 \%$ and $0.014 \%$, and between $0.002 \%$ and $0.003 \%$ for natal adult females. ${ }^{1}$ However, this estimation accounts for adults seeking treatment in specialty clinics, and is likely an underestimation of this population. ${ }^{1}$

Connected to the social determinants of health, research shows that the transgender population has an increased risk for sexually transmitted infections such as $\mathrm{HIV}_{1}{ }^{2}$ and mental health issues such as depression and suicide. ${ }^{3}$ Transgender individuals may also have unique healthcare needs, ${ }^{4}$ including hormone replacement therapy, and/ or gender reassignment surgeries in order to more closely align their bodies to their gender identity. These factors make it especially important for transgender individuals to be able to access adequate primary care in environments that are supported by appropriate policies and standards. Unfortunately, on both provider and policy levels, healthcare in Canada is not meeting the needs of the transgender population. ${ }^{4,5}$ Through reviewing current policies and guidelines, specific issues will be identified, and recommendations for how primary care providers can better support the transgender population will be highlighted.

Within the academicsetting, primary care providers are given little to no formal education on which to build a foundation of knowledge regarding transgender healthcare. ${ }^{6}$ This may discourage clinicians from providing services to transgender patients, as they are left in a position which requires them to take time to find resources and educate themselves on transgender healthcare. This is a critical area for change, as transgender individuals report that provider knowledge is an important component of their healthcare experiences that is currently lacking. ${ }^{4,7} \mathrm{~A}$ potential policy change would be to examine the current curriculum in medical schools and work to incorporate and/or strengthen a focus on gender diversity and transgender health in order to give clinicians a basic knowledge on which they can build. To date, a small number of Continuing Medical Education (CME) courses in transgender care have been offered in Canada, which provided both the opportunity for providers to gain knowledge, and incentive for them to obtain needed CME credits. One example of this type of CME course took place in Halifax in 2010, which was formatted as a day-long event focused on medical management of gender/sex transition. ${ }^{8}$ The implementation of similar CME courses across Canada would encourage providers to expand their knowledge in transgender care, and to present the information in formats that are accessible for primary care providers.

In order to provide clinicians with guidance in serving transgender patients, international Standards of Care have been developed by the World Professional Association of Transgender Health (WPATH). ${ }^{9}$ These standards cover transgender healthcare in a number of areas, including: care specifically within the primary care setting, gynecologic care, reproductive care, as well as mental health services. ${ }^{9}$ In addition, Sherbourne Health Centre in Toronto, Ontario has created its own guidelines for healthcare providers, which have been adapted throughout Ontario, and may be more relevant for Canadian providers. ${ }^{10}$ However, one noteworthy issue with existing standards of care is that they are merely guidelines that can be interpreted and $\mathbf{}$ 
applied differently within the healthcare setting. ${ }^{6}$ Thus, the standards may be difficult to implement for primary care providers who have no previous knowledge in transgender care. In order to effectively incorporate existing guidelines, healthcare organizations should ensure that health professionals are supported with adequate education and organizational policies around transgender care. Not only would this provide the institutional support that providers need when serving transgender patients, but it would also prevent current situations whereby transgender individuals are left waiting for healthcare services while policies are being developed at the organizational level. ${ }^{7}$

As transgender patients continue to present to primary care providers within the healthcare setting, it is increasingly important that they have knowledge in transgender health and are supported by policies that facilitate adequate care for this population. Through the creation of policies at governmental and organizational levels, primary care providers will be better equipped to serve transgender people, and to provide them with the same standard of care that many Canadians have come to expect.

\section{References}

1. American Psychiatric Association. Diagnostic and statistical manual of mental health disorders (5th ed.). Washington, DC: American Psychiatric Association; 2013.

2. Bauer GR, Travers R, Scanlon K, Coleman TA. High heterogeneity of HIVrelated sexual risk among transgender people in Ontario, Canada: A province-wide respondent-driven sampling survey. BMC Pub Health. 2012;12:292.

3. Goldblum P, Testa RJ, Pflum S, Hendricks M, Bradford J, Bongar B. The relationship between gender-based victimization and suicide attempts in transgender people. Prof Psychol Res Pr. 2013;43(5):468-75.

4. Bauer G, Hammond R, Travers R, Kaay M, Hohenadel KM, Boyce M. “I don't think this is theoretical; This is our lives": How erasure impacts health care for transgender people. J Assoc Nurses AIDS Care. 2009;20:348-61.

5. Snelgrove JW, Jasudavisius AM, Rowe BW, Head EM, Bauer GR. "Completely out-at-sea" with "two-gender medicine": A qualitative analysis of physicianside barriers to providing healthcare of transgender patients. BMC Health Serv Res. 2012;12:1472-6963.

6. Dewey J. Challenges of implementing collaborative models of decision making with trans-identified patients. Health Expect. [Internet]. 201319 Sept [cited 2014 March 18]. Available from: http://onlinelibrary.wiley.com. proxy.library.brocku.ca/enhanced/doi/10.1111/hex.12133/

7. Sperber J, Landers $S$, Lawrence $S$. Access to health care for transgender persons: Results of a needs assessment in Boston. Int J Transgend. 2005;8:75-91.

8. IWK Health Centre. Bridging the gaps in transgender healthcare: Medical management of gender/sex transition. 2010 June 11.

9. Coleman E, Bockting W, Botzer M, Cohen-Kettenis P, DeCuypere G, Feldman $J$, et al. Standards of care for the health of transsexual, transgender, and gender-nonconforming people, version 7. Int J Transgend. 2007;13:165-232.

10. Sherbourne Health Centre. Guidelines and protocols for comprehensive primary health care for trans clients. [Internet]. 2009 [cited 2014 March 9]. Available from: www.sherbourne.on.ca/PDFs/Trans-Protocols.pdf

\section{Katie Ross}

Katie is currently completing her Master's in Applied Health Sciences at Brock University. She is studying the factors that allow transgender individuals to have positive healthcare experiences in a deficits based healthcare system. 